\title{
Reflets
}

Revue ontaroise d'intervention sociale et communautaire

\section{Réflexions sur un projet d'entraide communautaire}

\section{Brigitte Malenfant}

Volume 3, numéro 1, printemps 1997

Enfance et familles en contexte d'appauvrissement

URI : https://id.erudit.org/iderudit/026149ar

DOI : https://doi.org/10.7202/026149ar

Aller au sommaire du numéro

Éditeur(s)

Reflets : Revue ontaroise d'intervention sociale et communautaire

ISSN

1203-4576 (imprimé)

1712-8498 (numérique)

Découvrir la revue

Citer ce document

Malenfant, B. (1997). Réflexions sur un projet d'entraide communautaire.

Reflets, 3(1), 16-28. https://doi.org/10.7202/026149ar

Tous droits réservés (C) Reflets : Revue ontaroise d'intervention sociale et communautaire, 1997

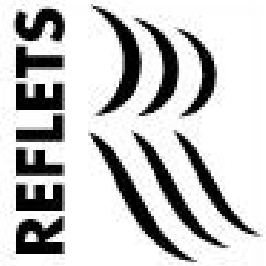

Ce document est protégé par la loi sur le droit d'auteur. L'utilisation des services d'Érudit (y compris la reproduction) est assujettie à sa politique d'utilisation que vous pouvez consulter en ligne.

https://apropos.erudit.org/fr/usagers/politique-dutilisation/ 


\section{Réflexions sur un projet d'entraide communautaire}

Au printemps 1996, un groupe de personnes d'Ottawa-Carleton s'est impliqué dans un projet pour identifier des solutions à la situation de pauvreté dans laquelle elles se trouvaient. Cette entrevue avec quelques participantes donne un aperçu des réalisations de ce projet et montre comment il a permis à un groupe de personnes de se prendre en charge, c'est-à-dire de retrouver une certaine forme d'autonomie et d'estime d'elles-mêmes. L'entrevue a été réalisée par Brigitte Malenfant.

Reflets: Carole, pourrais-tu nous décrire brièvement le projet d'entraide communautaire?

Carole: Le but du projet était premièrement de permettre aux assistés sociaux et aux assistées sociales d'identifier des solutions face à la pauvreté qui sévit en ce moment dans la région d'OttawaCarleton. L'autre but était de faciliter la mise en oeuvre du projet et de faire connaitre les solutions publiquement. On voulait aussi démystifier l'aide sociale, augmenter la concertation entre les organismes et entre les individus par rapport à ces problèmes. Un autre objectif visait à promouvoir l'autonomie des personnes à faible revenu. Hélène, as-tu quelque chose à ajouter?

Hélène: Je pense que le seul autre point c'était de donner la parole aux gens, de médiatiser les résultats et les solutions. On parle souvent des assistés sociaux dans les journaux mais rarement à partir de la perspective des personnes qui vivent la pauvreté.

Reflets: Est-ce que chacune aimerait se présenter brièvement, donner un portrait de sa situation, dire ce qui l'a conduite à se joindre à ce projet et parler de ses attentes par rapport à celui-ci. 
Patricia: Moi, ce qui m'a amenée à participer au projet c'est la recherche d'autonomie, de liberté personnelle, la possibilité de connaitre d'autres ressources, de prendre des choses en main. En même temps, en travaillant à ce projet, je savais que nous serions en contact avec d'autres organismes, d'autres personnes. Ainsi, on peut faire d'autres projets à partir de celui-là parce qu'on a connu des personnes qui peuvent avoir d'autres idées. De plus, au niveau économique, je savais que le projet m'aiderait à soulager les problèmes de ma famille.

Hélène: Je travaille dans un organisme qui vise la prise en charge personnelle et familiale du revenu, de la situation économique des individus. On travaille principalement avec les francophones d'Ottawa-Carleton.

Monique: Moi, je cherche à démolir les mythes reliés aux assistés sociaux.

Lise: Le projet m'a d'abord intéressée parce qu'il permettait de chercher des solutions économiques viables permettant de survivre. Dans le quartier où je vis, les gens ont de l'argent et trouver comment tu «fit» là dedans, c'est pas évident. C'était aussi pour rencontrer des gens.

Carole: Je suis la coordonnatrice du projet. Ce qui m'a incitée à m'y joindre, c'est que j'ai vécu beaucoup de pauvreté moi aussi. Et puis mon but était d'aider des personnes à retrouver leur autonomie et à s'en sortir.

Louise: Je trouvais le projet intéressant pour briser l'isolement et essayer de trouver des solutions.

Ce n'est pas en restant chez toi que tu arrives à quelque chose. Puis comme j'ai un côté fonceur, ambitieux, je ne peux pas rester enfermée. C'est impossible, je prends tout ce qui vient et puis j'essaie de m'en sortir.

Reflets: La prochaine question consiste à vous demander de décrire un peu comment le projet a débuté. Comment vous êtes-vous rencontrées? Dans quel projet chacune de vous est-elle impliquée?

Patricia: Moi, je participe à la boîte verte, c'est-à-dire l'achat en gros. Je participe aussi à un collectif de femmes immigrantes. 
Monique: Je participe aussi à l'achat en gros et à la cuisine collective et j'aime ça!

Louise: Je participe aussi à l'achat en gros. J'ai commencé à participer aux cuisines collectives l'année passée. C'est là que j'ai commencé à sortir de chez moi.Je fais aussi partie du groupe de soutien familial et du jardin communautaire. Ça m’a beaucoup aidée à reprendre confiance en moi.

Lise: Moi, je participe aux cuisines collectives et à l'achat en gros.

Patricia: Je pense que ce serait bien de regarder comment Carole a rejoint les gens et comment nous sommes venues la rencontrer.

Carole: On a fait de la publicité dans les journaux communautaires pour annoncer le projet, pour vérifier si les gens avaient le goût de faire le projet, si ils ou elles avaient des idées. Nous avons annoncé une rencontre publique. Il y a des gens qui sont venus et qui ont apporté eux-mêmes l'idée des jardins, des cuisines, etc. La première réunion a servi à discuter de projets possibles, ce qui était réalisable. Ensuite, le recrutement s'est fait de bouche à oreille. Je suis allée dans les centres communautaires deVanier, d'Overbrooke-Forbes et de la Basse-ville. C'est là que j'ai fait le plus de recrutement. J'ai ensuite fait d'autres présentations à R.E.A.C.H. (Centre de ressources, d'éducation pour personnes ayant un handicap) et à la Magie des lettres ainsi qu'à Cumberland.

Hélène: Dans chacune des communautés, il était important de trouver une personne qui vivait la situation de pauvreté. Cette personne faisait ensuite du bouche à oreille. Dans toutes les communautés, on a trouvé des bénévoles qui ont mis des affiches dans les buanderies, sur les poteaux téléphoniques et à d'autres endroits stratégiques. On se rend compte que ce qui fonctionne c'est vraiment le bouche à oreille, le contact personnel basé sur la confiance qu'ont les gens entre eux. Comme on connaissait le phénomène d'isolement, on savait que le bouche à oreille était le meilleur moyen. On n'avait pas prévu rencontrer des milliers de personnes. Notre objectif était de rejoindre entre 150 et 200 personnes si le projet obtenait un financement d'un an mais comme nous n'avons pas obtenu ce financement, nous avons réduit les objectifs. 
Lise: Oui, quand tu te trouves en situation de pauvreté, toutes les portes se ferment. Tu ne connais plus personne. Ça fait qu'il y a bien des endroits où tu ne vas pas parce qu'il faut des sous. Même s'il y a de la publicité, la pauvreté limite les endroits où les gens vont.

Reflets: J'imagine que les gens se sentent vulnérables et qu'il $y$ a peut-être une peur de rencontrer des personnes.

Lise: Peut-être pour certains, mais ça dépend des personnalités. Les personnes que tu as ici autour de la table ne sont pas des gens qui ont peur. Mais il y en a beaucoup d'autres qui ne se sont pas joints au projet et c'est peut-être ça finalement qui est au fond de l'affaire. Je me suis rendu compte qu'on parle des médias qui ont des préjugés contre les gens qui vivent de l'aide sociale. Mais il y en a aussi qui reçoivent un chèque d'aide sociale et qui ont des préjugés contre les autres prestataires.

Patricia: Ce sont les médias qui mettent le feu aux poudres. Je n'ai jamais entendu quelque chose de positif à la radio au sujet des assistés sociaux. Je suis entourée de personnes qui vivent la situation et je la comprends. Mon mari travaille mais notre revenu est quand même faible. Notre situation est un peu différente mais nous avons tout de même des problèmes économiques. C'est vraiment incroyable ce que j'entends à la radio. Comme immigrante, je trouve ça très dur. Les médias généralisent trop. On vit les mêmes problèmes que l'on soit Canadiens ou non. Je rencontre du monde dans l'autobus. Des gens me demandent, «est-ce que tu travailles?» Il me disent «les immigrants viennent voler notre travail... Bien, je leur dis, est-ce qu'il y en a du travail?»

Reflets: Au Nicaragua, comment est perçue la situation de pauvreté, est-ce que tu peux expliquer la différence avec ici peut-être?

Patricia: La pauvreté est traitée un peu différemment d'ici. Les pauvres ne sont pas rejetés comme tel. Ils ne se sentent pas isolés. Les gens qui ont de l'argent ont plutôt tendance à aider ceux qui en ont moins. 
Reflets: Mais au niveau des médias...

Patricia: Les médias ne parlent pas de ça. Il n'y a pas de programmes d'aide sociale. Ça parle de pauvreté mais c'est différent. Les médias parlent plutôt du fait que le gouvernement devrait mettre sur pied des programmes pour les enfants, pour les personnes âgées et les étudiants.

Reflets: Donc, tu viens d'une société où il y a plus d'entraide entre les gens. Crois-tu que cela a contribué à t'encourager à te joindre au groupe d'entraide ici?

Patricia: Dans ma culture, tu n'as pas besoin d'aller chercher quelqu'un; les gens viennent quand on a besoin parce que les autres vivent la même situation. Ici c'est difficile de trouver des gens. Chez nous les gens se sentent plus concernés par la situation des autres. Ils comprennent que les projets collectifs sont l'affaire de tout le monde, pas seulement de quelques personnes. Ici, je me rends compte que la jeunesse ne participe pas. Chez nous, ce sont souvent eux qui apportent les idées.

Reflets: Avant de revenir à votre situation, j'aimerais qu'on revienne à la question que vous avez abordée tout à l'heure, celle des gens qui n'ont pas participé au projet. Comment expliquez-vous que les gens qui sont dans le besoin ne participent pas à un projet de ce genre?

Monique: Moi,je connais beaucoup de personnes qui vivent de façon isolée. J'essaie de les tirer tranquillement. Par exemple, j'ai finalement convaincu ma mère de participer à la boîte verte. Ça m’a pris un mois et demi.

Reflets: Quelles sont les raisons qui l'empêchaient de participer?

Monique: Mes parents ont toujours travaillé. Mais mon père n'est plus capable de travailler et ils se sentent dépourvus. Il y a une sorte de fierté qui faisait que...

Lise: Moi, je pense que c'est une explication que tu ne trouveras pas dans un livre. C'est une question d'énergie. Quand tu vis la pauvreté, l'énergie est consacrée à la survie. Ça prend de l'énergie 
pour générer de l'énergie puis je pense qu'il y a beaucoup de ça dans l'affaire. Statistiquement, les gens les plus pauvres sont aussi les plus malades. La pauvreté est une sorte de cage où on s'enferme mentalement. Personne d'autre que soi-même ne peut nous aider à sortir de la pauvreté.

Carole: Moi, quand je vivais cette situation-là, les gens m'abaissaient. Je n'avais pas envie de sortir parce que je me faisais pointer du doigt. C'est déprimant, c'est bien décourageant et ça prend beaucoup de force pour t'en sortir. C'est bien difficile de se sortir du petit cocon qu'on se tisse.

Reflets: C'est comme une protection contre l'extérieur!

Patricia: J'aimerais faire un petit parallèle avec ce que Carole dit. C'est à peu près comme déménager au Canada. Il faut être fort et lutter pour s'en sortir et s'injecter dans la société. Alors c'est à peu près la même situation parce que ça prend beaucoup d'énergie pour se faire accepter par les autres. Il y a des problèmes semblables, celui du travail, de l'intégration à la société.

Hélène: Je pense que dans la culture francophone, on a souvent tendance à être passifs et à regarder les choses passer. Si je regarde mon rôle ici comme directrice à Entraide budgétaire, quand nous mettons un nouveau service sur pied, je réalise qu'il faut persévérer. Je pense aux ateliers d'impôts par exemple. La première année, il y avait trois personnes aux ateliers, la deuxième année c'était cinq personnes par atelier. Puis là, on est rendu à six ou sept, trois années plus tard. Le réseau fonctionne beaucoup de bouche à oreille. Quand tu as aidé trois personnes, tu sais que l'année prochaine tu en aideras cinq et ainsi de suite. Le danger c'est souvent que le financement est coupé parce qu'on n'a pas eu de résultats assez vite. Et puis je pense que dans le projet d'entraide, plusieurs des bénévoles étaient fatigués de travailler pour les autres. Quand le projet d'entraide a été créé et qu'on demandait «voulez-vous faire quelque chose», on l'a probablement perçu comme «voulez-vous faire quelque chose pour nous autres», pas nécessairement comme "voulez-vous faire quelque chose pour vous-mêmes». 
J'ai beaucoup d'espoir que nos ambassadeurs et nos ambassadrices pour la phase 2 sont les personnes rassemblées ici autour de la table et les autres qui ont vécu la phase 1. Quand les boîtes vertes sont sorties d'ici et que les gens sont arrivés chez eux avec ces boîtes vertes, les voisins ont dit "qu'est-ce que c'est que ça?» Alors les gens se disent "peut-être que nous autres aussi». Cet aspect visuel est important. Quand les gens n'ont pas d'argent, ils partent du principe que c'est pour les autres ces affaires-là parce qu'eux, ils ont de l'argent. Mais quand ils ou elles voient des gens de leur milieu qui ont la même situation socio-économique et qui profitent de ça, ils ou elles veulent participer. Alors c'est un peu de faire ses preuves et puis comme Lise a mentionné tantôt, c'est sûr que quand les gens recevant de l'assistance sociale ont eu des coupures de $22 \%$ en octobre 1995, beaucoup n'avaient plus d'argent pour la nourriture. Le budget coupé de $22 \%$, il faut encore payer le loyer. Ça reste la priorité et puis même de sortir et de prendre l'autobus pour venir chercher une boite verte, ce n'est pas très réaliste. C'est pourquoi le projet se voulait un projet de quartier, un projet de cuisine et puis je pense que tant qu'on n'arrivera pas à ce niveau là, on va avoir de la difficulté à recruter des gens pour participer parce que le déplacement est très difficile. Juste au point de vue financier, c'est très difficile.

Reflets: Quelles ressources (personnelles ou communautaires) le projet vous a-t-il permis de découvrir ou d'utiliser?

Patricia: Moi, personnellement,j'aime beaucoup participer au projet parce que ça m'a donné une place dans la société. J'ai réalisé que je viens d'ailleurs mais que $\mathrm{j}$ 'ai quand même pu apporter quelque chose, pour moi-même et aux autres. Je suis la coordonnatrice de la boîte verte.

Reflets: Et ça implique quoi exactement?

Patricia: Je suis responsable des finances. Je passe la commande. Je vais à la banque. Alors, moi, ça me valorise beaucoup, ça me donne confiance en moi. Je réalise que je peux aussi me débrouiller ailleurs. Ça me donne cette expérience qui me servira peut-être 
plus tard. Si j'aime ça, je vais donner mon énergie pour ça, mais j'apprends aussi quelque chose pour moi-même. Le partage avec les autres m'aide à connaitre et à accepter les autres. Ça m'aide à voir comment les gens réagissent dans ces situations. Ça m'aide aussi à connaitre des ressources où je peux aller... Comme hier, je ne connaissais pas Entraide budgétaire mais je sais que pour la prochaine déclaration d'impôts je viendrai ici. Personnellement, c'est une richesse, je découvre beaucoup de choses!

Monique: Moi, j'ai bien aimé le groupe d'achat en gros. Pour un petit montant tu reçois beaucoup. Et puis j'aime bien placer la nourriture dans les paniers avant que les gens viennent les chercher.

\section{Reflets: Comment fonctionne l'achat en gros?}

Lise: Au début du mois, tu fais ton paiement pour la nourriture que tu veux acheter. Nous avons choisi le début du mois parce que les assistés sociaux, les assistées sociales et les gens qui ont une pension reçoivent un chèque à ce moment. Alors, à la fin du mois ou à la troisième semaine, quand le frigo et l'armoire sont vides, il y a quelque chose qui entre là. La boîte verte arrive la troisième semaine. Au début du mois tu peux toujours t'acheter quelque chose, ce ne sont pas les deux premières semaines qui t'inquiètent mais la fin du mois.

Reflets: Le panier de nourriture dure une semaine?

Carole: Deux semaines, une semaine, selon la grosseur de la famille.

Hélène: À la première rencontre, les gens décident ce qu'ils veulent recevoir. Ils établissent une boîte standard pour simplifier la préparation des boîtes individuelles. Tout le monde ensemble a décidé que ce sont les fruits et légumes frais qu'ils utiliseront. C'est bien parce que dans les milieux pauvres, on ne trouve pas de produits frais à la fin du mois.

Lise: Moi, ce que j'ai découvert comme ressources, c'est quelque chose de personnel, lors de mon travail auprès de la communauté des gens d'affaires. Pour la cuisine collective, j'ai été impliquée dans la levée de fonds. Donc j'ai approché des gens de la communauté 
des gens d'affaires, les épiceries. Chez quelques-uns je savais que c'était peine perdue mais, il y en a d'autres qui ont été très ouverts. Nous voulions faire un repas communautaire au spaghetti donc nous voulions les ingrédients pour préparer le repas. Je suis allée voir les grossistes. Je dois dire que ça a été très positif. C'est surprenant avec la situation économique actuelle. Ça brise le mythe qui veut que tout le monde s'en moque.

Au point de vue personnel, c'est une reconnaissance de mon vécu, de mes acquis. J'ai choisi de m'orienter en communication. C'est mon domaine, c'est moi, ça m'appartient. Cette petite expérience là, ça a l'air bien insignifiant mais c'est vraiment prendre possession de qui je suis.

Carole: Ça m'a permis de constater qu'il y a encore de l'entraide qui existe. J'ai rencontré beaucoup de gens aux jardins communautaires. Il y avait bien des choses que je ne connaissais pas mais ces gens-là avaient des expertises. Ça m'a étonnée et même à eux, ça leur a fait du bien de me dire comment faire.

Lise: On se rend compte que le mythe qu'ils ou elles ne savent rien faire est très présent. C'est loin d'être vrai. Ces personnes là en ont des expertises comme Carole le disait.

Carole: Ah, oui, ils et elles en ont. J'étais fière d'eux au repas communautaire. Ils et elles sont pratiquement allés chercher tout ce dont on avait besoin pour faire le repas.J'ai trouvé ça extraordinaire, je vous lève mon chapeau...

Monique: Pour revenir sur ce qu'elle a dit. Mon mari s'est toujours fait dévaloriser. Il n'a pas d'éducation. Je l'ai encouragé à construire une table sur laquelle on prépare les boîtes vertes. Avec des bouts de bois, il a fait quelque chose d'utile. Maintenant, il a le goût de faire autre chose. Si quelqu'un lui donnait la chance de se prouver, il le ferait.

Louise: Moi, je n'ai pas toujours été dans cette situation. Avant je vivais assez bien. Mais en raison de certaines circonstances de la vie,j'ai dégringolé. Je connaissais beaucoup de choses mais j'ai perdu confiance en moi. C'est la cuisine communautaire qui a fait que je suis sortie. À la cuisine, on s'échange des services. Ça a commencé comme ça. Ensuite, j'ai un autre projet, puis un autre 
projet. Alors on s'ouvre, on reprend la place qu'on avait avant lorsque tout allait bien. Mais, si je n'avais pas téléphoné, je serais peut-être encore dans mon petit coin, parce que je ne sortais pas, je ne parlais à personne.

Lise: Mais il faut le courage de le faire, de sortir des idées préconçues, arrêter de se dire ça ne sert à rien, ça ne vaut pas la peine, qu'estce que je vais aller faire là.

Louise: On a pensé à des solutions dans le but de s'en sortir et puis qu'estce qu'on pouvait faire, puis la moitié des gens habitent des petits appartements où il n'y a pas d'espace pour jardiner. Nous avons obtenu un terrain dans la cour du Centre de ressources communautaires de la Basse-ville. Chacun et chacune à notre tour, on a planté, enlevé les mauvaises herbes, récolté et partagé les produits.

Reflets: Combien de personnes participaient?

Louise: On était sept. Nous avons commencé petit pour agrandir dans les années à venir.

Monique: Ma belle-famille et ma famille, on s'est mis ensemble puis on a fait le jardin. C'est une tradition que mon beau-père avait commencée.

Hélène: Le groupe d'entraide s'est réuni pour venir en aide à la cuisine collective, au jardin communautaire, au groupe d'échange. Ils sont allés chercher des fonds pour aider aux autres groupes à fonctionner. Ils ont réussi à écrire des lettres puis ils ont obtenu du financement. Ils ont pris ce financement-là et appuyé le jardin communautaire deVanier.Alors ça aussi, c'était intéressant comme démarche. C'est pas des gros montants d'argent, mais c'est pour dire que non seulement il y avait des circuits de familles qui travaillaient au jardin communautaire, il y avait un autre circuit de personnes qui travaillaient pour aller chercher de l'argent pour venir en aide à ces groupes-là. Il y a aussi eu des échanges de vêtements. 
Reflets: La prochaine question porte sur comment le projet vous a permis de vous prendre en charge. Je pense que ça revient peut-être à ce que vous avez déjà dit, je ne sais pas si vous avez autre chose à rajouter à ce sujet-là?

Louise: Ça m'a permis de devenir plus autonome. Avec le groupe de soutien, on voulait sortir, mais à trois ou quatre familles, c'était mieux. L'été dernier, nous sommes allés cueillir des fraises puis on a eu beaucoup de plaisir. Nous avons fait une bonne cueillette. Ensuite, on a fait de la confiture ensemble. Nous voulons faire d'autres activités, se regrouper ensemble.

Carole: Je dois dire qu'ils et elles ont pris l'initiative de faire cela euxmêmes. Je n'étais pas là. J'ai constaté leur autonomie.

Louise: On s'échange aussi de l'information. Par exemple, cet été, tout le monde disait "on aimerait ça sortir nous autres, aller à la campagne». Et puis un moment donné, on a eu une ressource. Quelqu'un qui nous a loué son chalet pour la modique somme de 5 par jour. Ça fait que je suis allée en vacances et j’en ai profité parce que la nature c'est bien important pour moi.

Reflets: Donc, ce que tu dis Carole, c'est que les membres du groupe acquièrent une certaine autonomie personnelle et de groupe. Ils dépendent de moins en moins de toi comme coordonnatrice d'un projet. Ils et elles prennent des initiatives.

Louise: Oui, puis on dirait que les portes s'ouvrent. Je prends tout ce qui passe et je vois que je vais m'en sortir.

Patricia: Pour répondre à comment on devient plus autonome, je vais parler de la cuisine collective. Le projet de la cuisine collective a commencé en mai cette année et devait se terminer en novembre 1996. Mais on ne veut pas que ça finisse alors le groupe a eu l'idée de faire un dîner communautaire. Ça a bien fonctionné. Moi je disais, on peut faire un autre dîner communautaire plus tard, mais ça va être des échanges. On fera des recettes aussi de notre pays pour que les gens goûtent de la nourriture différente.

Hélène: La levée de fonds sert à acheter les choses importantes comme les herbes, les épices qui peuvent être très dispendieuses. Il faut aussi acheter des chaudrons. Je pense à un autre point important qui 
est l'appui communautaire. Les gens passaient devant un centre francophone tout le temps mais est-ce qu'ils seraient entrés dans l'édifice? Les gens ont osé demandé. Ils ont obtenu la cuisine qui n'était pas beaucoup utilisée. On nous a même dit que nous pouvons revenir n'importe quand.

Patricia: Moi je dirais que l'union fait la force. Nous obtenons quelque chose du centre francophone et eux ont une visibilité. C'est un échange.

Lise: J'aimerais ajouter quelque chose. Au début je me disais que je finirais par voir une différence en ce qui concerne mon budget. J'ai vu la différence tout de suite le même mois!

Reflets: Une fois qu'ils ont reçu le panier, est-ce que les gens ont de la difficulté à le gérer, à ne pas perdre de nourriture ou est-ce que dans la plupart des cas, ça c'est bien passé?

Carole: Ça c'est bien passé. Je pense qu'ils se sont arrangés pour garder leurs légumes. Certains les ont blanchis. D'autres en congèlent.

Patricia: Moi,j'ai invité le voisin pour qu'il participe. On a fait des échanges de légumes. Ça me fait sentir comme chez-moi.

Reflets: En terminant, comment entrevoyez-vous votre avenir?

Monique: Moi, mon but c'est que ma fille de 13 ans ne vive pas ce que j'ai vécu.

Patricia: Moi je ne travaille pas. Il n'y a que mon mari qui travaille. Je suis étudiante et mon travail à la maison n'est pas tellement reconnu parce qu'il ne rapporte pas d'argent.Alors dans ce sens, je me sens bien parce que, même si je ne rapporte pas d'argent à la maison, la cuisine collective me permet d'obtenir la nourriture dont nous avons besoin. Maintenant, mon mari sait qu'on a de la nourriture dans le frigo et il est très content. Il me dit «je garde les enfants et tu vas travailler à la cuisine collective». C'est ma façon de soulager les problèmes économiques dans ma famille. 
Reflets: Est-ce que tu penses que ça a changé les conditions de vie de ta famille de façon générale.

Patricia: Oui, bien c'est sûr, quand mon conjoint est venu chercher la boîte verte, il était content. Je n'ai pas un travail rémunéré mais ça me donne une autonomie. Je contribue aussi. Ca soulage les problèmes économiques et ça réduit la tension.

Hélène: À partir des cuisines collectives, il y a eu le désir de se former une coopérative de travail. C'est du développement économique.Après les avoir vues à l'oeuvre, je n'ai aucun doute que cette coopérativelà fonctionnera.

Lise: Oui, il y a un avenir individuel mais il y a aussi un avenir collectif pour nos projets.

Reflets: Je crois que nous pouvons nous arrêter ici. Je voudrais vous remercier au nom de l'équipe de Reflets et en mon nom personnel. J'aimerais bien voir la salle où préparez vos boîtes vertes et la table construite par le mari de Monique.

\section{Note}

Les personnes intéressées à obtenir plus de détails au sujet de ce projet peuvent s'adresser à Entraide budgétaire au (613) 746-0400. 